\title{
L'hyperactivité de l'adénosine désaminase dans les globules rouges
}

\section{Les nouvelles de ce numéro ont été préparées par : J.-C. Dreyfus A. Kahn}

De nombreux travaux ont été consacrés au déficit en adénosine désaminase (ADA), associé habituellement aux déficits immunologiques combinés. On connaît moins le syndrome d'hyperactivité de l'ADA, décrit en 1970 par Paglia et al. Il consiste en une augmentation considérable ( 50 à 70 fois) de l'activité de l'ADA, confinée aux globules rouges. Tous les autres tissus explorés ont une activité normale, y compris fibroblastes et lymphoblastes en culture. Le catabolisme excessif de l'adénosine a pour conséquence son indisponibilité pour la synthèse des nucléotides à adénine, d'où résulte une anémie hémolytique sévère. L'affection est transmise comme un caractère autosomique dominant. Outre les cas américains [1] elle a été décrite en France [2] et au Japon [3]. On ignore la base moléculaire de cette hyperactivité localisée. Par tous les tests disponibles, l'ADA synthétisée paraît normale, et la quantité de protéine immunoréactive est parallèle à l'activité. On ne sait donc si la lésion moléculaire se situe dans le gène, au niveau de la transcription, de la traduction, ou dans la stabilité du messager ou de la protéine. Chottinger et al. [4] (Ann Arbor, USA) ont tenté de répondre à ces questions. Ils ont d'abord vérifié la conformité des résultats actuels avec les données antérieures : activité 75 fois supérieure dans les globules rouges de malades que dans des globules rouges normaux, avec augmentation du même ordre du taux de la protéine ADA, mais pas d'élévation dans une lignée lymphoblastoïde. Ils ont ensuite examiné le niveau du gène : les Southern blots se sont montrés normaux, éliminant l'hypothèse de remaniements majeurs et d'amplification génique. Plus instructive fut l'analyse de l'ARN messager par Northern blot: en effet l'ARN, de taille $1,5 \mathrm{~kb}$, a la même concentration dans les réticulocytes des malades et dans ceux de sujets à niveau comparable de réticulocytes sans élévation de l'ADA ; les quantités de messager dans les lymphoblastes du malade et d'un témoin sont également du même ordre.

Enfin, la mesure de l'efficacité de traduction en système acellulaire a également été effectuée. Les ARN polyadénylés du malade et d'un témoin riche en réticulocytes ont été traduits en système de réticulocytes de lapin en présence de méthionine marquée, et l'ADA précipitée par son antisérum, puis dissoute en milieu dissociant, soumise à électrophorèse et autoradiographie. La traduction du messager de l'ADA se fait sous forme d'une bande de 42000 daltons, spécifiquement éliminée par l'addition d'ADA non marquée. A peine visible chez le témoin, cette bande est intense chez le malade.

La conclusion des auteurs est que l'anomalie se situe au niveau posttranscriptionnel, et consiste en un accroissement de l'efficacité de la traduction. Reste à comprendre d'une part la cause première de ce changement, et l'on devra pour cela attendre le clonage et le séquençage de l'ADN complémentaire de l'ADA et, d'autre part et surtout, la sélectivité tissulaire. Les auteurs invoquent l'existence possible d'un inhibiteur dans les globules normaux ; ils s'appuient sur la présence, dans les réticulocytes normaux, d'une quantité notable d'ARN messager de l'ADA, qui reste inefficace dans la traduction. Cet inhibiteur serait absent chez les malades ou plus probablement, puisqu'il s'agit d'une affection dominante et donc de sujets hétérozygotes, il y aurait une mutation dans le gène de l'ADA qui rendrait le messager insensible à l'inhibiteur. 